\title{
CIGRE-Session 2014
}

\section{G. Christiner}

Online publiziert am 6. November 2014

(C) Springer Verlag Wien 2014

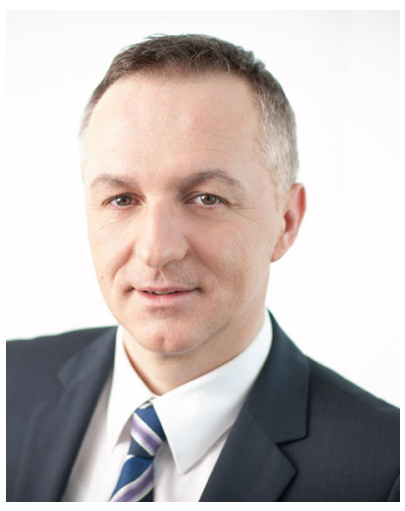

VDir. Dipl.-Ing. Mag. (FH) Gerhard Christiner
Die 45. CIGRE-Session fand vom 24. bis 29. August 2014 in Paris statt. Über 3.200 Session-Teilnehmer aus 90 Ländern, darunter 74 aus Österreich, sowie 8.500 Besucher der parallel zur Session stattfindenden Ausstellung unterstrichen die Brisanz der aktuellen Entwicklungen im Bereich der Elektrizitätswirtschaft.

Als weltweit bedeutendste Plattform ihrer Art bietet die CIGRE-Session alle zwei Jahre Vertretern der Energiewirtschaft und Industrie, Elektrotechnik-Experten und Wissenschaftlern die Gelegenheit zum Erfahrungs- und Wissensaustausch sowie zur Diskussion aktueller Entwicklungen im Bereich der Energiewirtschaft.

Die Schwerpunktthemen 2014 waren unter anderem:

- Electricity markets and regulation

- Insulated cables

- HVDC and power electronics

- Information systems and telecommunications

- Protection and automation
- System operation and control

- System development and economics

Die voranschreitende Strommarktintegration in Europa sowie die Veränderungen in der europäischen Erzeugungsstruktur stellen die europäischen Stromnetze vor große Herausforderungen. Das Eröffnungs-Panel stand daher auch unter dem Titel "Challenges of the transition in power systems - regional perspectives".

In den Poster- und Plenarsessions wurden auf Basis von 315 präsentierten Papers die Einzelaspekte dieser großen Systemveränderungen diskutiert. Auch in diesem Jahr kamen wieder acht Beiträge aus Österreich. Sie können diese auf den folgenden Seiten nachlesen.

Ich möchte diese Gelegenheit auch dazu nutzen, meinem Kollegen Herbert Lugschitz sehr herzlich zur Verleihung des "Distinguished Member Awards 2014" zu gratulieren. Kollege Lugschitz ist seit der Schaffung des Awards im Jahr 1996 erst der dritte österreichische Vertreter, der sich diese besondere Auszeichnung aufgrund seines großen Einsatzes innerhalb der Organisation über viele Jahre hinweg verdient hat.

Ich wünsche Ihnen eine spannende Lektüre und darf Sie schon jetzt auf die CIGRE-Session 2016 hinweisen, die vom 21. bis zum 26. August stattfinden wird.

Mag. (FH) Dipl.-Ing. Gerhard Christiner

Vorsitzender des Österreichischen Nationalkomitees der CIGRE
Christiner, Gerhard, Austrian Power Grid AG, Wagramer Straße 19, 1220 Wien, Österreich (E-Mail: gerhard.christiner@apg.at) 